\title{
Controle de dopagem de anabolizantes: o perfil esteroidal e suas regulações
}

\author{
Marlice Aparecida Sipoli Marques ${ }^{1,2}$, Henrique Marcelo Gualberto Pereira ${ }^{1}$ \\ e Francisco Radler de Aquino Neto ${ }^{1}$
}

\section{RESUMO}

O conceito de perfil esteroidal é discutido neste artigo. As principais vias biossintéticas são apresentadas. A importância do monitoramento do perfil esteroidal é demonstrada dentro da clínica médica e da medicina esportiva. Parâmetros da literatura para a identificação de dopagem por esteróides endógenos são apresentados, assim como os fatores que acarretam alterações no perfil esteroidal normal. É dada atenção especial a essa última abordagem.

Palavras-chave: Esteróides. Perfil. Biossíntese. Dopagem.

\section{ABSTRACT \\ Control of doping with anabolic agents: the steroid pro- file and its regulations}

The concept of steroid profile is discussed in this paper. The main metabolic routes are presented. The importance of evaluating steroid profiles is demonstrated, with special attention to clinical medicine and sports. Parameters used

1. LABDOP, Instituto de Química, Departamento de Química Orgânica, Universidade Federal do Rio de Janeiro.

2. LABDOP, Instituto de Química, Departamento de Química Analítica, Universidade Federal do Rio de Janeiro.

Recebido em 13/11/01

2a versão recebida em 15/6/02

Aceito em 1/11/02

Endereço para correspondência:

Francisco Radler de Aquino Neto

Laboratório de Controle de Dopagem (LABDOP-LADETEC)

Centro de Tecnologia, Bloco A, sala 512 - Instituto de Química

Universidade Federal do Rio de Janeiro

21949-900 - Rio de Janeiro, RJ - Brasil

Tel.: 5521 2562-7489, fax: $55212562-7489$

E-mail: radler@iq.ufrj.br in the literature for doping control of endogenous steroids are briefly evaluated, as well as the factors responsible for alterations in the normal steroid profile. Special focus is turned to the latter approach.

Key words: Steroids. Profile. Biosynthesis. Doping.

\section{DEFINIÇÃO DE PERFIL ESTEROIDAL}

$\mathrm{O}$ termo perfil esteroidal foi empregado pela primeira vez em meados da década de 60. Em 1968, Horning ${ }^{1}$ publicou um artigo sobre o perfil esteroidal baseado na análise cromatográfica de esteróides neutros.

De acordo com o dicionário Webster ${ }^{2}$, a palavra perfil (profile) originou-se do latim em 1656 e pode ser definida como um conjunto de dados que, representados de forma quantitativa, na forma de gráfico, mostra a tendência do parâmetro avaliado, independente da técnica de detecção ${ }^{2}$. Alguns pesquisadores podem inferir que essa definição é muito ampla, já que, erroneamente, pode-se pensar que para estabelecer o perfil esteroidal é necessário coletar todos os dados disponíveis no cromatograma relativo aos esteróides detectados ${ }^{3}$.

\section{BIOSSÍNTESE E REGULAÇÃO DE ESTERÓIDES}

Os hormônios corticossupra-renais de ocorrência natural consistem em moléculas de esteróides sintetizadas e liberadas pelo córtex supra-renal. A secreção dos esteróides corticossupra-renais é controlada pela liberação hipofisária de corticotropina (ACTH). O córtex supra-renal libera grande número de esteróides na circulação. Alguns possuem atividade biológica mínima e atuam primariamente como precursores, enquanto outros ainda não tiveram sua função estabelecida. Os esteróides hormonais podem ser classificados em esteróides que possuem efeitos importantes sobre o metabolismo intermediário (glicocorticosteróides), esteróides que exercem principalmente atividade de retenção de sal (mineralocorticosteróides) e os que exibem atividade estrogênica ou androgênica, os quais são os objetivos deste trabalho. 


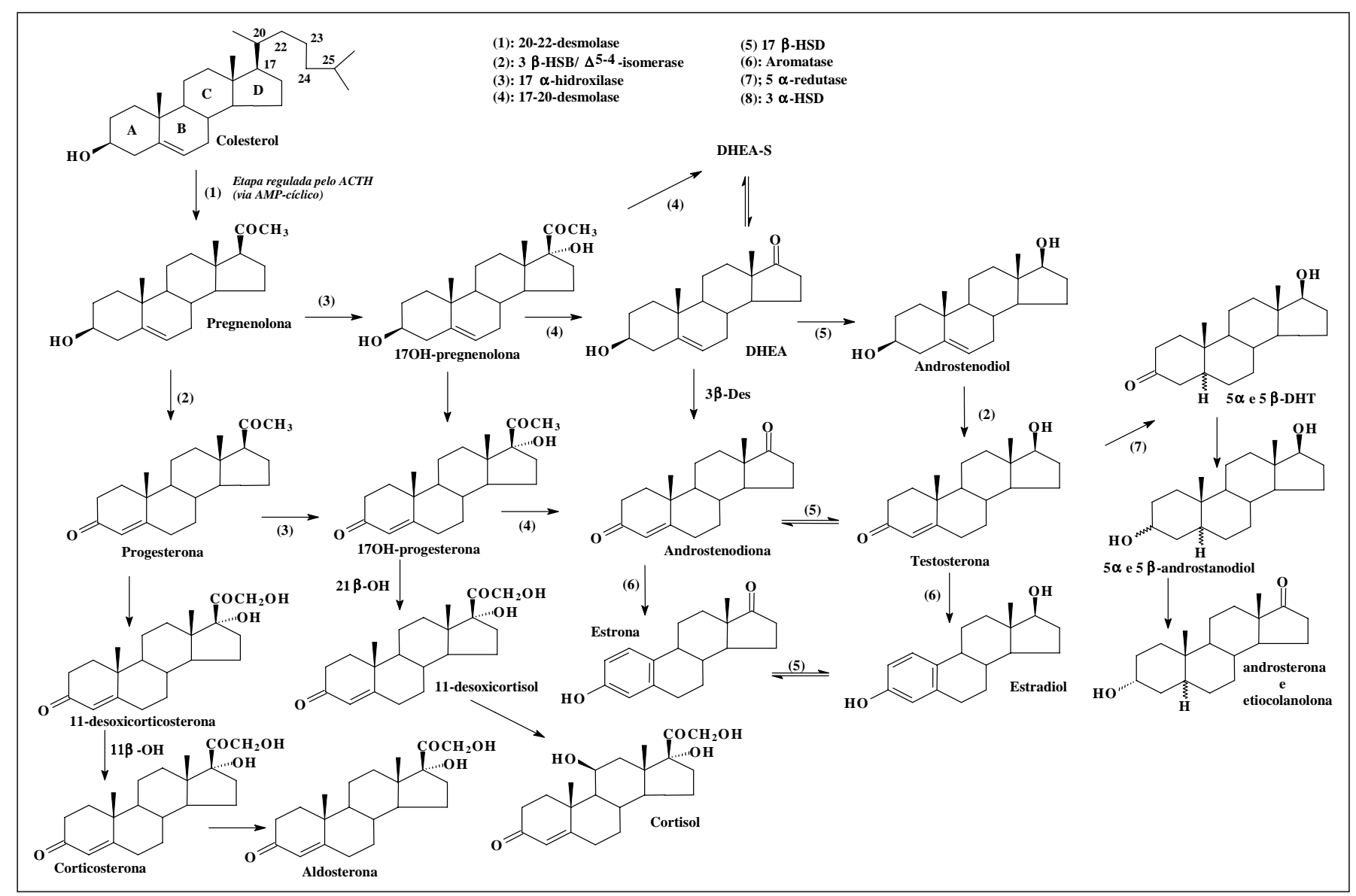

Fig. 1 - Biossíntese e metabolismo dos esteróides endógenos ${ }^{1,3-6}$

A figura 1 ilustra, de forma simplificada, a biossíntese e o metabolismo dos esteróides endógenos no organismo humano ${ }^{1,3-6}$.

\section{HORMÔNIO ADRENOCORTICOTRÓPICO (ACTH): REGULAÇÃO NA BIOSSÍNTESE E METABOLISMO DOS ESTERÓIDES ENDÓGENOS}

O ACTH é um hormônio que estimula o córtex da suprarenal a sintetizar cortisol (glicocorticosteróide), corticosterona, aldosterona (mineralocorticosteróide) e várias substâncias androgênicas fracas (figura 2) ) $^{4,6}$. A formação da pregnenolona através da clivagem oxidativa da cadeia lateral do colesterol é o principal processo da esteroidogênese regulada pelo ACTH (figuras 1 e 2). Essa etapa é limitadora de taxa na seqüência de reações que levam à formação de hormônios esteroidais adrenais. Além disso, o ACTH aumenta a ligação do colesterol ao citocromo $\mathrm{P}_{450}{ }^{7,8}$. Esses fatos, juntamente com a evidência de que a disponibilidade de colesterol é o fator que limita a extensão da reação de clivagem do colesterol na mitocôndria intacta ${ }^{9}$, sugerem que o ACTH, via AMP cíclico, estimula a reação inicial de esteroidogênese do mesmo, tornando o substrato disponível quando há maior concentração da enzima dentro das mitocôndrias. O ACTH também atua aumentando a disponibilidade do colesterol, estimulando sua captação de lipoproteínas plasmáticas ${ }^{10}$.

Do ponto de vista quantitativo, a desidroepiandrosterona (DHEA) é o principal androgênio, visto que são secretados cerca de $20 \mathrm{mg}$ por dia (em parte na forma de sulfato). Todavia, tanto a DHEA como a androstenodiona são androgênios muito fracos ${ }^{11}$.

Nos seres humanos a testosterona $(\mathrm{T})$ é o androgênio mais importante secretado pelos testículos (figuras 1 e 3). As vias de síntese da $\mathrm{T}$ nos testículos são semelhantes às descritas para a supra-renal. No homem são produzidos diariamente cerca de $8 \mathrm{mg}$ de T. Cerca de $95 \%$ pelas células de Leydig e apenas $5 \%$ pelas supra-renais. O testículo também secreta pequenas quantidades de outro androgênio potente, a diidrosterona (DHT), bem como androstenodiona (AEDION) e desidroepiandrosterona (DHEA), que são androgênios fracos ${ }^{12-14}$. A principal via de degradação da T nos seres humanos encontra-se ilustrada na figura 1 . No 


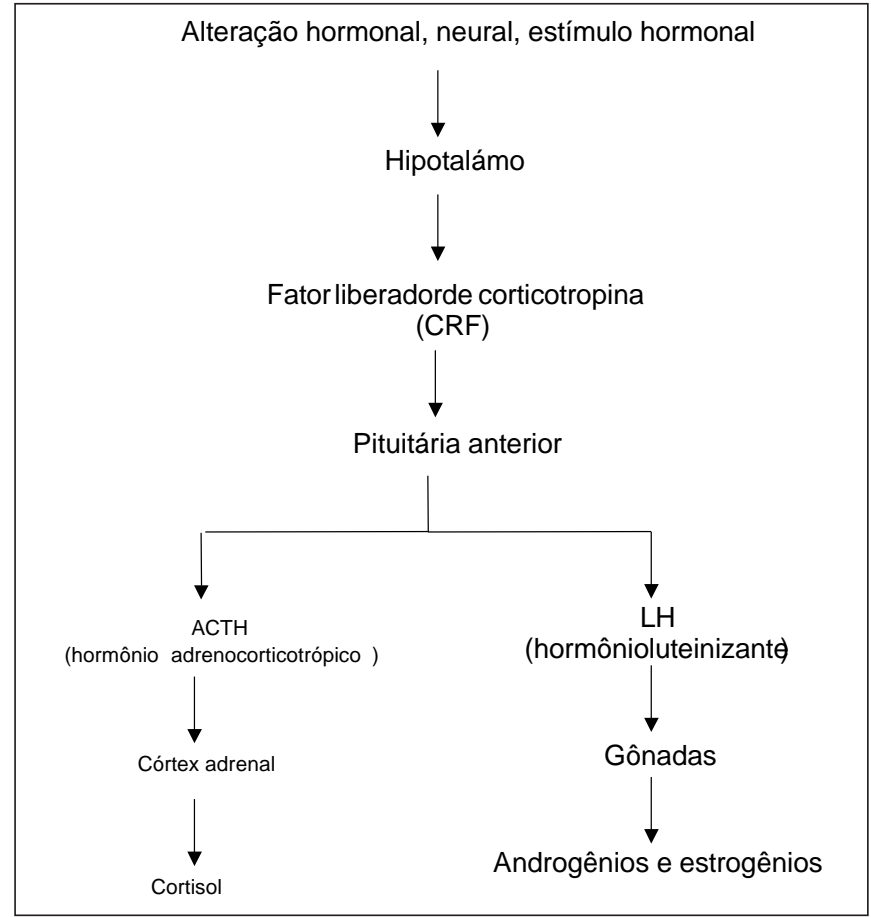

Fig. 2 - Controle hipotálamo-pituitária-adrenal sobre a secreção dos hormônios adrenais e gonadais ${ }^{15}$ fígado, a redução da ligação dupla e cetona do anel A, conforme observado com outros esteróides com configuração $\Delta^{4}$-cetona, resulta na produção de substâncias inativas, como androsterona (A) e etiocolanolona (E), que são posteriormente conjugadas e excretadas na urina ${ }^{15}$.

\section{ESTERÓIDES ENDÓGENOS DE BIOSSÍNTESE DESCONHECIDA}

\section{Norandrosterona (NA)}

A nandrolona (ND) é um dos anabolizantes mais encontrados em dopagem de atletas nos últimos anos ${ }^{16,17}$. No organismo humano a ND é metabolizada a norandrosterona (NA) e noretiocolanolona (NE, figura 4$)^{18-21}$, que são excretadas na urina na forma glicuconjugada. A administração dos precursores da ND, como a norandrostenodiona (NDA) e norandrostenodiol (NDOL), também leva à formação dos mesmos metabólitos (NA e NE, figura 5) ${ }^{17,20}$.

O principal metabólito da ND é a NA, apresentando a maior concentração na urina e a mais longa excreção (figura 6). A fim de excluir a possibilidade de produção endógena $^{22-29}$, o Comitê Olímpico Internacional (COI) estipulou concentração de corte (cut off) de $2 \mathrm{ng} / \mathrm{mL}$ e $5 \mathrm{ng} / \mathrm{mL}$

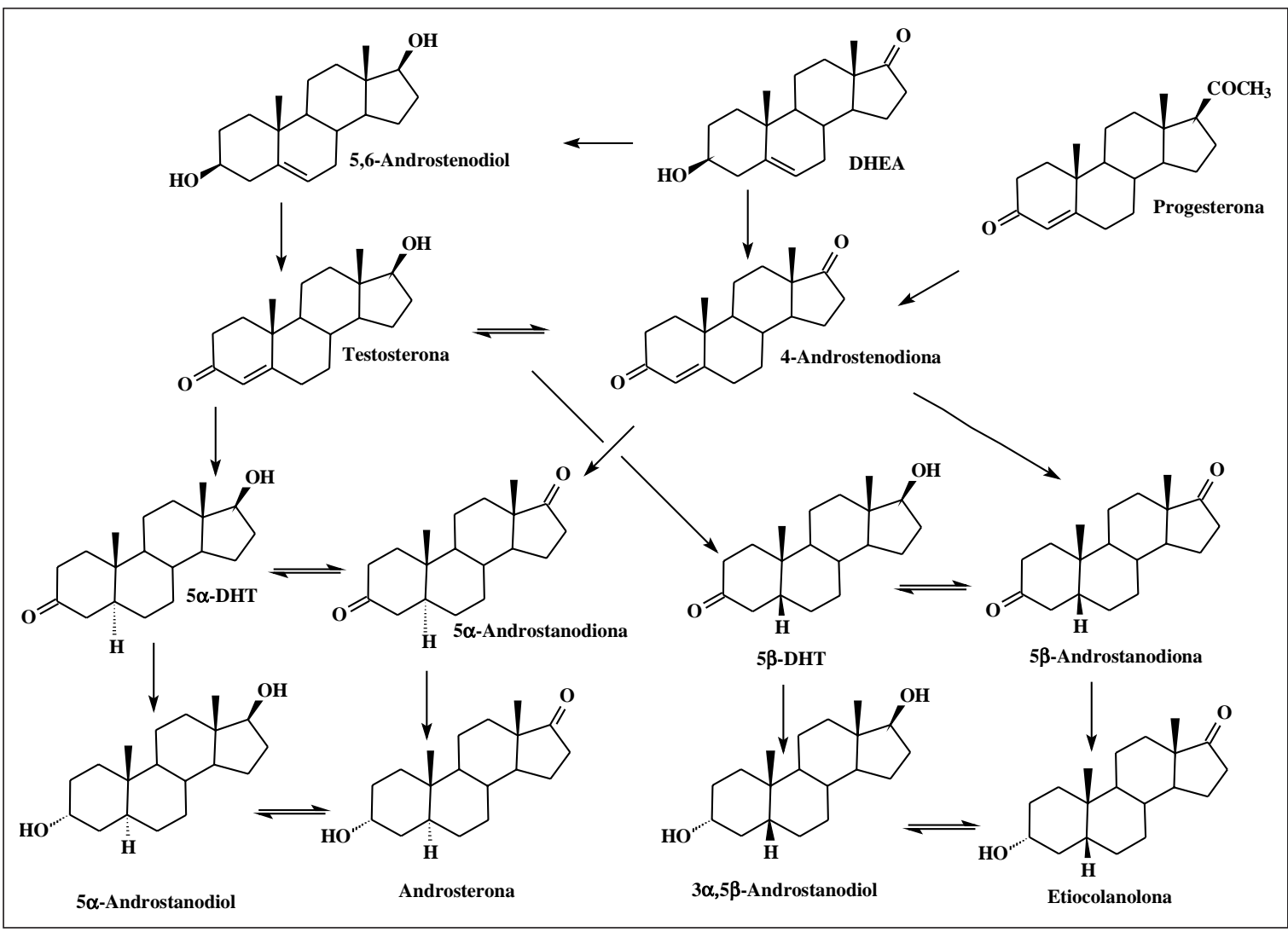

Fig. 3 - Metabolismo da Testosterona e do DHT 


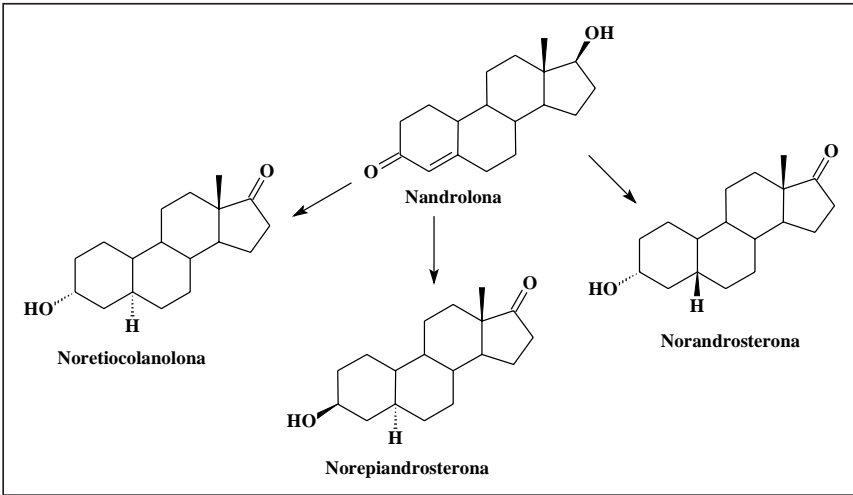

Fig. 4-Metabolismo da 19-Nortestosterona (nandrolona $)^{21,24,34}$

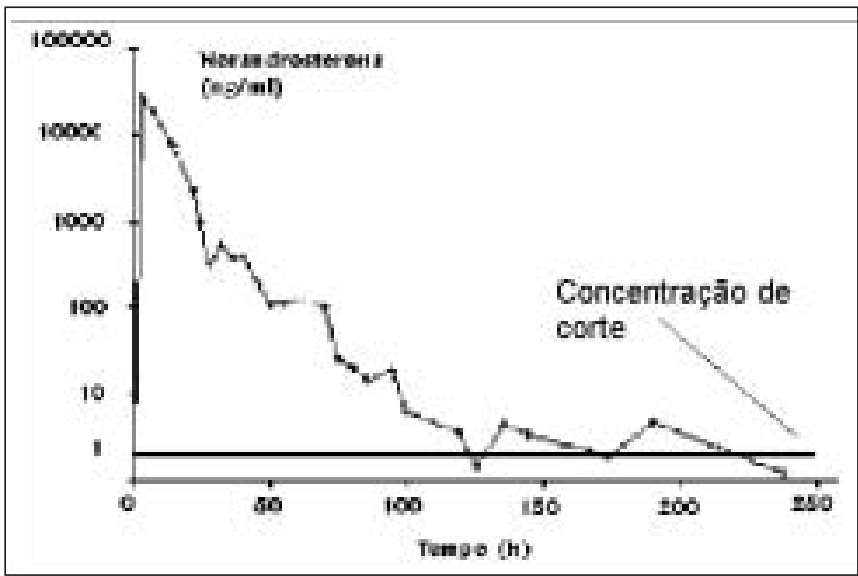

Fig. 6 - Curva de excreção de NA após a administração de 20mg de nandrolona ${ }^{34}$

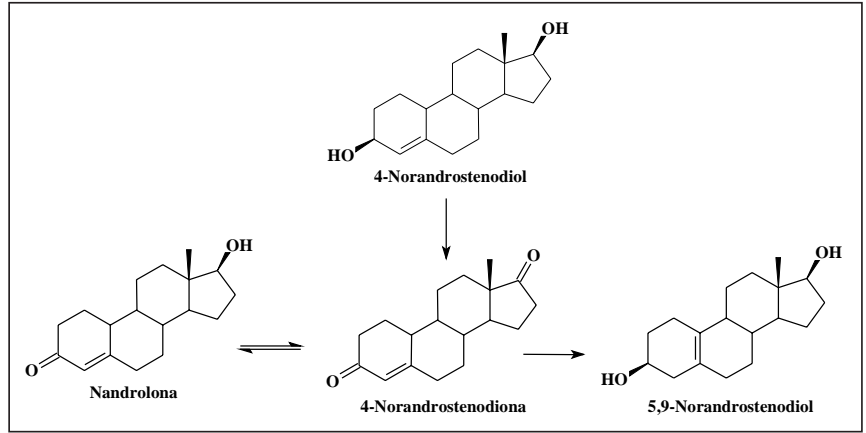

Fig. 5 - Metabolismo dos precursores da nandrolona ${ }^{34}$

para homens e mulheres, respectivamente, a ser considerada como um positivo do uso de ND, já que a mesma foi caracterizada com estudos em animais ${ }^{22-24}$ e humanos ${ }^{25-29}$. Abaixo dessas concentrações, até o presente momento, não se sabe a via de biossíntese endógena desse 19-nor-esteróide.

\section{IMPORTÂNCIA DO MONITORAMENTO DO PERFIL ESTEROIDAL}

Os esteróides de interesse no estabelecimento das condições normais e fisiopatológicas do homem podem ser reunidos em vários grupos distintos (tabela 1) ${ }^{1,3-6}$. Os hormônios esteroidais podem ser classificados em precursores e metabólitos. Os percussores e alguns dos seus metabólitos asseguram a função biológica (hormonal), sendo que a determinação desses contribuiria na avaliação a fun-

TABELA 1

Esteróides de interesse na avaliação do perfil esteroidal|,3-19a

Esteróides

Hormônios esteroidais precursores

Cortisol

Progesterona

DHEA

Pregnenolona

metabólitos

Androstenodiona

Diidrotestosterona

a: DHEA: Desidroepiandrosterona.

\section{Metabólitos e principal forma de eliminação}

Glicuconjugado

Tetrahidrocortisona, tetrahidrocortisol, $5 \alpha$-tetrahidrocortisol, tetrahidroaldosterona

17-OH-prtogesterona

Androstenodiona, androstenodiol

17-OH-pregennolona, progesterona, 17-OH-progesterona

Glicuconjugado

Testosterona, $6 \beta-\mathrm{OH}$-androsterona e $6 \beta-\mathrm{OH}$-etiocolanolona

$5 \alpha$ e $5 \beta$-androstanodiol, androsterona e etiocolanolona 


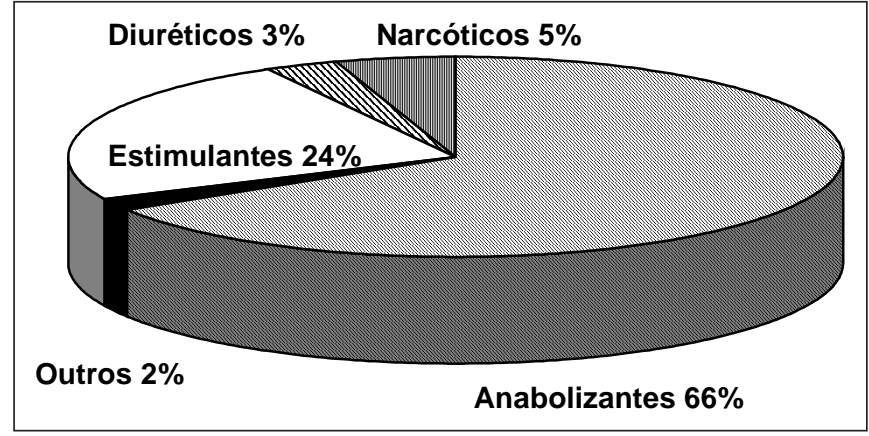

Fig. 7 -Percentagem de positivos identificados no controle de dopagem pelo COI (1986-1997) $)^{38}$

ção endócrina. Já a determinação dos principais metabólitos auxilia na elucidação dos erros biossintéticos.

Os esteróides precursores são biossintetizados nas glândulas e lançados na corrente sanguínea. Os metabólitos terminais são excretados na urina e bile, na forma livre e/ou conjugada ${ }^{3,30}$.

Shackleton ${ }^{31}$ e Donike et al..$^{32}$ foram os primeiros a reportar na literatura a elucidação do perfil esteroidal com o intuito de caracterizar dopagem em atletas pelo uso indevido de esteróides endógenos. Os autores descreveram as alterações provocadas nas quantidades relativas de um esteróide ou de seus diferentes metabólitos após a administração de alguns esteróides endógenos. Atualmente, distúrbios nessas razões são amplamente empregados na caracterização de dopagem por esteróides endógenos.

\section{ESTERÓIDES ANABÓLICOS E GLICOCORTICOSTERÓIDES}

\section{Esteróides anabólicos}

Esteróides anabólicos (EA) são substâncias relacionadas ao hormônio sexual masculino, a T (figuras 1 e 3) ${ }^{33,34}$. Esse hormônio exerce diversos efeitos no homem, inclusive o de aumentar a massa muscular e o peso corpóreo.

Nos esportes, os EA são administrados em atletas justamente com o intuito de aumentar a massa muscular e, conseqüentemente, garantir melhora no desempenho. $\mathrm{O}$ uso ilícito de EA iniciou-se na década de 50, entre levantadores de peso e fisiculturistas, tendo-se alastrado para outras modalidades esportivas. Devido a razões de ordem ética e aos efeitos nocivos à saúde, essas substâncias tiveram o uso proibido pelo COI a partir de 1976, na Olimpíada de Montreal, onde foi realizado pela primeira vez o controle de anabolizantes. Seis atletas foram punidos pelo uso indevido ${ }^{35}$.

A figura 7 permite visualizar a proporção relativa do uso dessas substâncias em relação às demais classes farmaco-

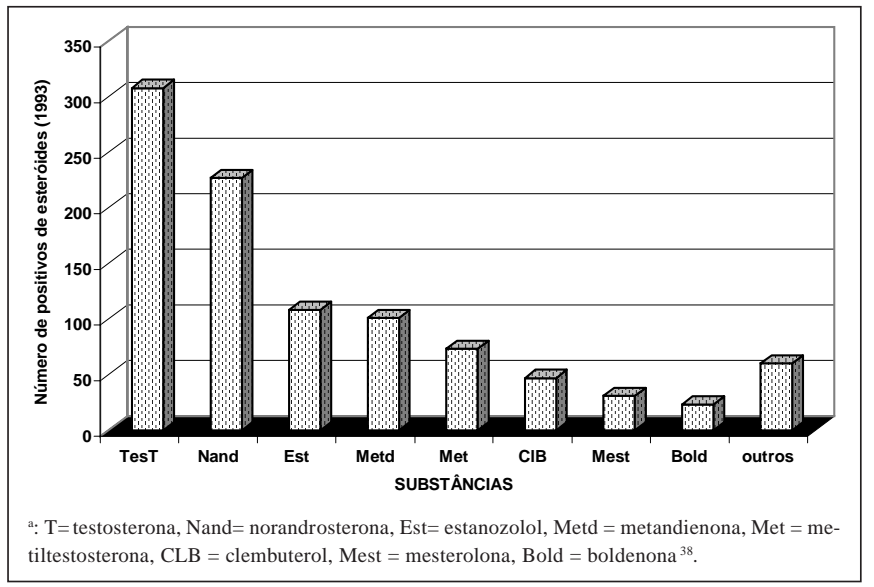

Fig. 8 - Estatística das substâncias anabolizantes ${ }^{a}$ esteroidais detectadas nos laboratórios olímpicos no ano de 1993 (COI $)^{38}$

lógicas de uso proibido para atletas ${ }^{36}$, nos últimos anos. Uma estatística do próprio COI (figura 8), no decorrer do ano de 1993, permitiu constatar que o esteróide mais empregado na dopagem de atletas era a $\mathrm{T}^{36}$.

\section{Glicocorticosteróides}

Glicocorticosteróides são substâncias de estrutura química relacionada ao hormônio cortisol conhecidas como esteróides adrenais. O cortisol exerce um papel importante no equilíbrio eletrolítico e no metabolismo de carboidratos, proteínas e lipídeos e possui potente efeito antiinflamatório ${ }^{37}$.

\section{PARÂMETROS DA LITERATURA PARA A IDENTIFICAÇÃO DE DOPAGEM POR ESTERÓIDES ENDÓGENOS}

A confirmação de esteróides endógenos em dopagem de atletas é, atualmente, um dos maiores problemas do controle. Por isso, é necessário encontrar critérios para discriminar o uso exógeno da produção endógena. Para tanto, utilizam-se, freqüentemente, parâmetros de referência (baseados na população européia) ${ }^{12,31,32,38-40}$ descritos a seguir.

\section{Desidroepiandrosterona (DHEA)}

Após a administração de DHEA (figura 1) ocorre aumento na concentração da A, E, $3 \alpha, 5 \alpha$-androstenodiol $(3 \alpha, 5 \alpha-$ DIOL) e DHEA. Esses são os parâmetros mais estáveis na avaliação da administração exógena de DHEA (se a dopagem for recente). Uma suspeita de positivo ocorre quando a concentração estiver acima do limite superior da faixa de referência dos valores normais da população em estudo, ou seja, $10 \mathrm{ng} / \mathrm{ml}^{31,41-43}$. 


\section{Diidrotestosterona (DHT)}

A administração exógena de DHT, que é o metabólito ativo da T, leva a aumento da excreção da própria DHT e de seus metabólitos (figuras 1 e 8) ${ }^{12,32,42,43}$. Entretanto, AEDION e epitestosterona (EpiT) não são influenciadas. Conseqüentemente, o abuso da aplicação de DHT pode ser caracterizado pelo aumento das razões entre a DHT e alguns esteróides endógenos (tabela 2 e figura 3 ).

\section{$5 \alpha$-androstanodiona (ADION)}

Após a administração de ADION, o perfil esteroidal assemelha-se ao observado quando se administra DHT (figura 8 , tabela 3). A única diferença é que o aumento observado nos valores de concentração dos metabólitos da DHT é inferior aos valores obtidos após a administração de DHT, porém superior aos valores normais estipulados para a população em estudo. Não se observa aumento na excreção da $5 \alpha$-androstanodiona na urina após a sua administração ${ }^{12}$.

\section{Androsterona (A)}

Administração de A leva a aumento na sua própria concentração excretada, assim como na do $5 \alpha, 3 \alpha$-DIOL. Não se observam modificações significativas nas concentrações de T e EpiT formadas ${ }^{12}$.

\section{Androstenodiona (AEDION)}

A administração exógena de AEDION pode ser caracterizada por aumento nas concentrações de A, E, T e, conseqüentemente, na razão $\mathrm{T} / \mathrm{EpiT}^{9,43-45}$. Além da presença dos metabólitos $6 \alpha-\mathrm{OH}$-androstenodiona, $6 \beta-\mathrm{OH}$-androsterona, $6 \beta-\mathrm{OH}$-etiocolanolona e $6 \beta-\mathrm{OH}$-epiandrosterona.

\section{Testosterona (T)}

A T e a EpiT são os únicos endógenos que possuem um critério estabelecido pelas confederações esportivas e pelo COI. Uma razão T/EpiT > 6 na urina de um atleta é consi-

\begin{tabular}{lcc}
\hline \multicolumn{3}{c}{$\begin{array}{c}\text { TABELA 2 } \\
\text { Parâmetros aceitos para a confirmação } \\
\text { de um positivo de DHT' }\end{array}$} \\
\hline \multicolumn{1}{c}{ Parâmetro } & Homem & Mulher \\
DHT & $>20 \mathrm{ng} / \mathrm{ml}$ & $>18 \mathrm{ng} / \mathrm{ml}$ \\
A/E & $>2,9$ & $>2,1$ \\
$5 \alpha, 3 \alpha-\mathrm{DIOL} / 5 \beta, 3 \alpha-\mathrm{DIOL}$ & $>1,5$ & $>1,3$ \\
DHT/E & $>8,2$ & $>8,5$ \\
DHT/EpiT & $>0,73$ & $>2,3$ \\
\hline
\end{tabular}

a: $5 \alpha, 3 \beta$-DIOL, $3 \alpha, 5 \alpha$-androstanodiol; $5 \alpha, 3 \alpha$-DIOL, $3 \alpha, 5 \alpha$-androstanodiol; DHT, diidrotestosterona; EpiT, epitestosterona; T, testosterona; A, androsterona e E, etiocolanolona. derada como dopagem, caso não seja comprovado que o mesmo possua as suas funções fisiológicas e/ou endocrinológicas alteradas ${ }^{46-52}$.

\section{Epitestosterona (EpiT)}

A possível administração simultânea de T e EpiT para fraudar o critério anterior levou tanto o COI como as confederações esportivas à proposição de positivo de EpiT, caso a mesma seja encontrada em uma concentração acima de $200 \mathrm{ng} / \mathrm{mL}^{16,27}$.

\section{5-androstenodiol (AEDIOL)}

A administração exógena de AEDIOL (figuras 1 e 9), segundo Schänzer et al., leva a aumento na concentração de testosterona e seus metabólitos ${ }^{42,44,45,50,51,53}$.

\section{Nandrolona (ND)}

Conforme já mencionado, o COI estabeleceu as concentrações de corte de $2 \mathrm{ng} / \mathrm{mL}$ e $5 \mathrm{ng} / \mathrm{mL}$ para homens e mulheres, respectivamente, como parâmetro para a confirmação de administração exógena de $\mathrm{ND}^{27}$. O problema dessa estratégia é mais complexo para as mulheres, em que uma série de parâmetros deve ser considerada. Por exemplo, é perfeitamente possível encontrar o metabólito NA na urina de mulheres grávidas, já que a ND está presente no fluido do folículo ovariano e na placenta durante a gravidez ${ }^{28,51,54-}$ 56. Nesses casos, além da concentração de corte, recomenda-se a realização da dosagem de gonadotropina coriônica humana (hCG), de modo a determinar a ocorrência ou não de gestação. Também é comum a observação da presença de NA em urina de mulheres que fazem uso do anticoncepcional norestisterona (noresteróide) ${ }^{27}$. Porém, nesse caso, a presença dos outros metabólitos provenientes da noretisterona permite excluir a possibilidade de um falso-positivo por ND.

\section{FATORES QUE ACARRETAM ALTERAÇÕES NO PERFIL ESTEROIDAL NORMAL}

Conforme descrito acima, alguns parâmetros interferem no perfil esteroidal normal ${ }^{31,37,57-60}$.

\section{Efeito de administração exógena de esteróides endó-} genos

Phillips (1996) ${ }^{60}$ estudou a relação, no soro, entre DHEA$\mathrm{S}$, AEDION e hormônios sexuais do homem e da mulher (figuras 1 e 2). O autor concluiu que, no homem, o nível de DHEA-S não pode ser correlacionado com o de testostosterona-glicuconjugada (T-G) ou testosterona-livre (T-L), mas é correlacionado, fortemente, com o da AEDION. Na mulher, a concentração de DHEA-S está relacionada não so- 


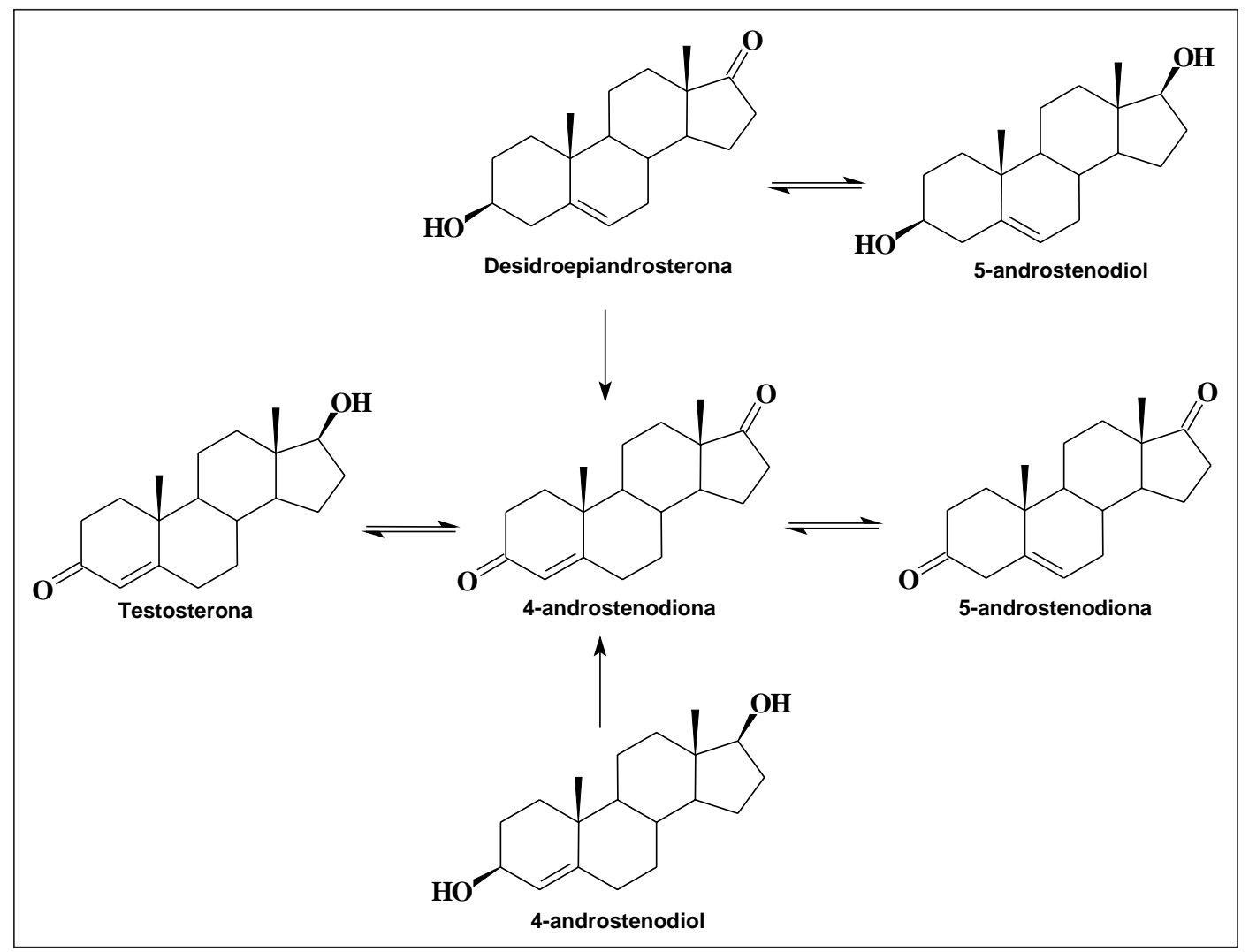

Fig. 9 - Metabolismo dos pró-hormônios da testosterona ${ }^{63}$

mente com a AEDION, mas também com a T e T-L. A DHEA$S$ não mostrou qualquer correlação com o estradiol e a insulina, tanto no homem quanto na mulher. A falta de correlação entre DHEA-S e T, no homem, é consistente com a biossíntese independente desses hormônios pela glândula adrenal e testículos, respectivamente.

Kazlauskas (1998) $)^{41}$ estudou o efeito da administração de DHEA (100 e 200mg, na forma de ésteres) por via oral, em indivíduo saudável. $\mathrm{O}$ autor concluiu que a concentração de DHEA aumentava duas-três vezes em relação ao valor normal apenas por um período curto (0-6 horas). $\mathrm{O}$ autor constatou também que havia aumento significativo na razão $\mathrm{A} / \mathrm{E}$ e $3 \alpha, 5 \alpha$-androstenodiol $/ 3 \alpha, 5 \beta$-androstenodiol ( $3 \alpha, 5 \alpha$-TDIOL/ $3 \alpha, 5 \beta$-TDIOL). A razão T/EpiT foi afetada apenas nas primeiras 10 horas após a administração, mas não excedeu significativamente os valores normais. Caso a amostra seja coletada após um período de 50 horas da administração da DHEA, esses valores podem ter retornado aos normais ou ainda algumas razões podem estar até invertidas, como, por exemplo, A/E. Portanto, o tempo em que a amostra foi coletada após a administração do fármaco é de fundamental importância na interpretação dos resultados. Os autores concluem ainda que, no caso de um positivo de DHEA, devem-se comparar os dados obtidos com os valores de referência da população em estudo ${ }^{41}$.

Efeito da administração de esteróides exógenos

A administração de esteróides exógenos diminui a secreção de esteróides endógenos por um mecanismo de retroalimentação negativa ${ }^{4,37}$. Estudos comprovam que a metandienona (um anabolizantes sintético) leva a diminuição na excreção de esteróides endógenos ${ }^{46,61}$. $\mathrm{O}$ uso prolongado de estanozolol leva a diminuição da razão A/E $\mathrm{E}^{46,61}$.

\section{Administração de álcool}

A ingestão de álcool, em altas doses, pode elevar a razão T/EpiT e diminuir a razão A/T através do aumento da excreção de T-conjugada e decréscimo na excreção de androsterona-conjugada. Essas alterações estão relacionadas à presença de etanol na urina ${ }^{61}$.

\section{Administração de probenicida e diuréticos}

A administração de probenecida reduz a excreção dos esteróides conjugados. As razões entre os esteróides não se modificam. Esse efeito é devido à inibição competitiva com os ácidos orgânicos pelos mecanismos de transporte ativos nos túbulos proximais dos rins ${ }^{32}$. 
Ao contrário da probenecida, os diuréticos não reduzem a excreção de esteróides; essas substâncias aumentam o fluxo de urina e, conseqüentemente, reduzem a concentração dos esteróides nessa excreta.

\section{Administração de finasterida}

A administração de finasterida leva à inibição da atividade da enzima $5 \alpha$-redutase, conseqüentemente, a diminuição considerável na razão $\mathrm{A} / \mathrm{E}$, na razão $3 \alpha, 5 \alpha$-DIOL/ $3 \alpha, 5 \beta$-DIOL e na concentração de $\mathrm{DHT}^{49}$.

\section{Efeito do estresse físico na biossíntese e metabolismo de esteróides endógenos}

É de conhecimento geral que a produção de hormônios pelas gônadas e pela supra-renal é controlada pelo eixo hipotálamo-pituitária (figura 2). Portanto, é de esperar que o estresse físico possa alterar não só a produção de ACTH, como também a do hormônio luteinizante (LH) ${ }^{4,37}$.

Yap et al. (1996) ${ }^{39}$ avaliaram os efeitos do estado emocional, neural e hormonal sobre o perfil de esteróides endógenos, após o estresse físico. Para tanto, os autores realizaram um estudo sistemático, em que os atletas foram divididos em três grupos, sendo um quarto grupo usado como controle. Com exceção dos grupos controles, os atletas foram submetidos a condições predeterminadas de treinamento, em que os parâmetros de umidade, ventilação, capacidade respiratória, consumo de oxigênio e treinamento intensivo foram avaliados.

Os autores avaliaram principalmente o efeito do estresse físico sobre o aumento do LH e da T. A análise foi realizada por cromatografia gasosa acoplada à espectrometria de massas (CG-EM). Uma avaliação das concentrações de T (figura 5) e LH e das razões de T/LH comprovou que, apesar de o estresse estimular a produção do LH, a concentração de $\mathrm{T}$ não era alterada significativamente quando comparada com os dados obtidos com o grupo controle. Conseqüentemente, ocorria diminuição da razão T/LH. Os autores destacam ainda a importância de realizar esse tipo de estudo em amostras de urinas coletadas em intervalos de tempo determinados, não devendo ser colhida a urina diurna, pois inversões nessas razões podem ser observadas.

Aumento do ACTH, por qualquer causa (estresse ou outros estímulos), pode levar à ocorrência de aumento de todos os precursores produzidos nas adrenais, tais como pregnenolona $\rightarrow$ 17OH-pregnenolona $\rightarrow$ DHEA (hormônios adrenais, figura 1). De acordo com a figura 2, tais fatores, além de aumento no ACTH, podem também levar a acréscimo na liberação do LH. Esse fato irá refletir-se em aumento da secreção dos hormônios adrenais e gônadas.

Resumindo, os parâmetros baseados na distribuição do perfil esteroidal de uma dada população são muito está- veis e devem ser empregados como valores de referência na confirmação de dopagem pelo atleta com esteróides endógenos ${ }^{3,32,46-49,60,61}$, com exceção da nandrolona. Esses parâmetros podem ser influenciados, contudo, pela administração de esteróides endógenos ao atleta, bem como de alguns esteróides exógenos ou fármacos como finasterida, tamoxifeno, probenecida, diclofenaco, diuréticos, etanol, trimetropima, entre outros. Atividade bacteriana também pode causar alterações desses parâmetros ${ }^{3,62}$. Portanto, técnicas de detecção que permitam a discriminação tanto da administração exógena de endógenos e a influencia desses parâmetros no perfil esteroidal normal viriam auxiliar na confirmação do abuso de esteróides endógenos ${ }^{63-69}$. Atualmente, uma nova abordagem no controle dessas substâncias vem sendo empregada. $\mathrm{O}$ enfoque consiste no emprego da CG-EM convencional para triagem dos supostos positivos e posterior confirmação por cromatografia gasosa/combustão acoplada à espectrometria de massas por razão isotópica (CG/C/EMRI).

\section{AGRADECIMENTOS}

Ao Conselho Nacional de Desenvolvimento Científico e Tecnológico (CNPq).

Todos os autores declararam não haver qualquer potencial conflito de interesses referente a este artigo.

\section{REFERÊNCIAS}

1. Horning EC, editor. Gas phase chromatography steroids. New York: Springer, 1968.

2. Webster on line dictionary. http://www.upgr.com.br/ dicionario.html. 2001.

3. Ruth S. Referenzbereich von urinaren steroidkoncentrationen und steroidquotienten - ein beitrag zur interpretation des steroidprofiles in der routinedopinganalytik. Dissertation, Deutsche Sporthochschule Koln, 1994.

4. Haynes Jr. RC, Murad F. Hormônios adrenocorticotrópicos; Esteróides adrenocorticais e seus análogos sintéticos; inibidores da biossíntese adrenocortical de esteróides. In: Goodman LS, Gilman AG, editors. As bases farmacológicas da terapêutica. Rio de Janeiro: Guanabara Koogan, 1987; 943-55.

5. Venturelli E, Cavarelli A, Secreto G. Methods for urinary testosterone analysis. J Chromatogr B 1995;671:363-8.

6. Wu FCW. Endocrine aspects of anabolic steroids. Clin Chem 1997;43: 1289-92.

7. Bell JJ, Harding BW. The acute action of adrenocorticotropic hormone on adrenal steroidogenesis. Biochim Biophys Acta 1974;348:285-98.

8. Gann DS, Dallman MF, Engleland WC. Reflex control and modulation of ACTH and corticosteroids. Int Rev Physiol 1981;24:157-99.

9. Kahnt FW, Milan A, Steffen H, Neher R. The rate-limiting step of adrenal steroidogenesis and adenosine 3',5'-monophosphate. Eur J Biochem 1974;44:243-50

10. Gwynne JT, Mahaffee D, Brewer HB, Ney RL. Adrenal cholesterol uptake from plasma lipoproteins; regulations by corticotropin. Proc Natl Acad Sci USA 1976;73:4329-33. 
11. Pradal MGP, Di Dio R. Andrógenos adrenais: DHEA e DHEA-S. http:/ /www.medicmail.com.br/PESQTEXT/DHEA.html/ANDR2GENOS, 1999.

12. Geyer H, Schanzer W, Schindeler U. Changes of the urinary steroid profile after sublingual application of dihydrotestosterone. In: Donike M, Geyer H, Gotzmann A, Mareck-Engelke U, Hauth S, editors. Recent advances in doping analysis (3). Köln: Sport und Buch Strauß 1996; 215-30.

13. Donike M. Steroid profiling in cologne. In: Donike M, Geyer H, Gotzman A, Rauth S, editors. Proceedings of the 10th Cologne Workshop on Dope Analysis, 7th to 12th of June 1992. Köln: Sport und Buch Strau $\beta$ 1993;47-68.

14. Palonek E, Garle M. Single injection of testosterone to seven volunteers: results from this study. In: Donike M, Geyer H, Gotzmann A, Mareck-Engelke U, Hauth S, editors. Proceedings of the 10th Cologne Workshop on Dope Analysis, 7th to 12nd of March 1992, Köln: Sport und Buch Strauß 1992;131-40.

15. Schänzer W, Donike M. Metabolism of anabolic steroids in man: synthesis and use of reference substances for identification of anabolic steroid metabolites. In: Donike M, Geyer H, Gotzmann A, Mareck-Engelke U, Hauth S, editors. Proceedings of the 10th Cologne Workshop on Dope Analysis, Köln: Sport und Buch Strauß 1993;97-100.

16. Merode A. Criteria to be applied to the quantitation and confirmation of anabolic steroids. COI Guidelines, 1998;8.

17. Mathurim JC, Herrou V, Bourgogne E, Pascaud L, Ceaurriz J. Gas chromatography-combustion-isotopic ratio mass spectrometry analysis of 19nortestosteroids: application to the detection of a nandrolone metabolite in urine. J Chromatogr B 2001;759:267-75.

18. Dehennin L, Bonnaire Y, Plou, Ph. Urinary excretion of 19-norandrosterone of endogenous origin in man: quantitative analysis by gas chromatography-mass spectrometry. J Chromatogr B 1999;721:301-7.

19. Robinson N, Taroni F, Saugy M, Ayotte C, Mangin P, Dvorak J. Detection of nandrolone metabolites in urine after a football game in professional and amateur players: a basian comparison. Forensic Sci Int 2001; 122:130-5.

20. Schänzer W, Donike M. Metabolism of anabolic steroids in man: synthesis and the use of reference substances for identification of anabolic steroid metabolites. Anal Chim Acta 1993;275:23-48.

21. Uralets VP, Gillette PA, Latvan RK. In: W. Schänzer, H. Geyer, A. Gotzmann, U. Mareck-Engelke, editors. Occurrence of 19-nordehydro-androsterone/etiocolanolone in nandrolone positive specimens. Recent advances in doping analysis. Köln: Sport und Buch Strauss 1996;35-42.

22. Martin AMG, Marino JIM, Tiedra GMP, Marabe JJR, Loscos MJC, Marino MM. Determination of nandrolone and metabolites in urine samples from sedentary persons and sportmen. J Chromatogr B 2001;761: 229-36.

23. Maghuim-Rogister G, Bosseloir P, Gaspar P, Dasnois G, Pelzer G. Determination of nandrolone in cattle. Ann Med Vet 1988;132:4437-40.

24. Gaillard Y, Vayssette F, Bailland A, Pepim G. Gas chromatographic-tandem-mass spectrometric determination of anabolic steroids and their esters in hair application in doping control and meat quality. J Chromatogr B 1999;735:189-205.

25. Carrasquilla MH. Gas chromatography-mass spectrometry analysis of anabolic compounds in bovine hair: evaluation of hair extraction procedures. Anal Chem Acta 2001;434:59-66.

26. Kintz P, Criminelli V, Ludes B. Doping control for nandrolone using hair analysis. J Pharm Biomed Anal 2001;24:1125-30.

27. Dehenin L, Bonnaire Y, Plou P. Urinary excretion of 19-norandrosterone of endogenous origin in man: quantitative analysis by gas-chromatography-mass spectrometry. J Chromatogr B 1999;721:301-7.
28. Lê bizec B, Monteau F, Gaudim I, André F. Evidence for the presence of endogenous 19-norandrosterone in human urine. J Chromatogr B 1999; 723:157-72.

29. Saugy M, Robinson N, Cardis C, Schweizer C, Rivier L, Mangim P, Ayotte C, Dvorak J. Nandrolone metabolites in football players: utility for in and out of competitions tests. In: Schänzer W, Geyer H, Gotzmann A, Mareck-Engelke U, editors. Recent advances in doping analysis. Köln: Sport und Buch Strauss 1999;95-107.

30. Nozaki O. Steroid analysis for biomedical diagnosis. J Chromatogr A 2001;935:267-78.

31. Shackleton CHL. Profiling steroid hormones and urinary steroids. J Chromatogr 1986;379:91-156.

32. Donike M, Rauth S, Wolansky A. Reference ranges of urinary endogenous steroids determined by GC-MS. In: Donike M, Geyer H, Gotzmann A, Mareck-Engelke U, editors. Proceedings of the 10th Cologne Workshop on Dope Analysis, Köln: Sport und Buch Strau $\beta$ 1993;69-86.

33. Shahidi NT. A review of the chemistry, biological action, and clinical applications of anabolic-androgenic steroids. Clin Ther 2001;23:135590.

34. Schänzer W, Delahaut P, Geyer H, Machnik M, Horning S. Long-term detection and identification of metandienone and stanozolol abuse in athletes by chromatography-high-resolution mass spectrometry. J Chromatogr B 1996;687:93-108.

35. Aquino Neto FR. O papel do atleta na sociedade e o controle de dopagem no esporte. Rev Bras Med Esporte 2001;7:138-48.

36. Schanzer W. (http://www.dopinginfo.de), 2001.

37. Goldfien A. Adrenocorticosteróides e antagonistas corticossupra-renais. In: Katzung BG, editor. Farmacologia básica e clínica. Rio de Janeiro: Guanabara Koogan, 1998;450-61.

38. Mareck-Engelke U, Geyer H, Donike M. Stability of steroid profiles. In: Donike M, Geyer H, Gotzman A, Marek-Engelke U, Rauth S, editors. Proceedings of the 10th Cologne Workshop on Dope Analysis. Köln: Sport und Buch Strauß 1993;87-9.

39. Yap BK, Kaslaukas R, Elghazi K, Johnston GAR. Profiling of urinary testosterone and luteinizing hormone in exercise-stressed male athletes, using gas chromatography-mass spectrometry and enzyme immunoassay techniques. J Chromatogr B 1996;687:117-25.

40. Waasdorp TJA. Evaluation of the T/LH ratio. In: Donike M, Geyer H, Gotzmann A, Mareck-Engelke U, Hauth S, editors. Proceedings of the 10th Cologne Workshop on Dope Analysis, 7th to 12nd of March 1992. Köln: Sport und Buch Strau $\beta$ 1992;123-4.

41. Kazlauskas R. Effects of dehydroepiandrosterone on urinary steroids. In: Schänzer W, Geyer H, Gotzmann A, Mareck-Engelke U, editors. Proceedings of the 15th Cologne Workshop on Dope Analysis. Köln: Sport und Buch Strau $\beta$ 1998;83-90.

42. Urallets VP, Gillette PA, Lavten RK. Over-the-counter anabolic steroids 4-androsten-3,17-dione; 4-androsten-3b,17b-diol, and 19-nor-4-androsten-3,17-dione; excretion studies in men. In: Schänzer W, Geyer H, Gotzmann A, Mareck-Engelke U, editors. Proceedings of the 17th Cologne Workshop on Dope Analysis. Köln: Sport und Buch Strauß 1999;26-36.

43. Ueki M, Okano M, Ikekita A. Nagano strategy against natural hormone doping, testosterone, DHT, androstenedione and DHEA. In: Schänzer W, Geyer H, Gotzmann A, Mareck-Engelke U, editors. Proceedings of 16th Cologne Workshop on Dope Analysis. Köln: Sport und Buch Strauss 1999;145-6.

44. Shänzer W, Breidbach A, Geyer H, Kuk CV, Nolteernsting E. Metabolism of nortestosterone, norandrostenedione and norandrostenediol: identification of 3a,16a-dihydroxy-5a-estran-17-one glucuronide and sulphate. In: Donike M, Geyer H, Gotzman A, Marek-Engelke U, Rauth S, editors. Proceedings of the 18th Cologne Workshop on Dope Analysis. Köln: Sport und Buch Strauß 2000;155-76. 
45. Geyer H, Mareck-Engelke U, Schänzer W. The analysis of nutritional supplements for anabolic-androgenic steroids. In: Schänzer W, Geyer H, Gotzmann A, Mareck-Engelke U, editors. Proceedings of 18th Cologne Workshop on Dope Analysis 2000. Köln: Sport und Buch Strauss 2000;33-41.

46. Mareck-Engelke U, Geyer H, Schänzer W. The interpretation of female steroid profiles In: Schänzer W, Geyer H, Gotzmann A, Mareck-Engelke U, editors. Proceedings of the 15th Cologne Workshop on Dope Analysis. Köln: Sport und Buch Strau $\beta$ 1998;51-70.

47. De Boer D, Waasdorp TJA, Schoone P, Thijssen JHH. The power of the ketoconazole test. In: Donike M, Geyer H, Gotzman A, Marek-Engelke $\mathrm{U}$, Hauth S, editors. Proceedings of the 10th Cologne Workshop on Dope Analysis. Köln: Sport und Buch Strauß 1994;105-10.

48. Mareck-Engelke U, Geyer H, Donike M. Stability of steroid profiles (3): The circadian rhythm of urinary ratios and excretion rates of endogenous steroids in male. In: Donike M, Geyer H, Gotzmann A, MareckEngelke U, Hauth S, editors. Proceedings of the 12th Cologne Workshop on Dope Analysis. Köln: Sport und Buch Strauß 1995;121-37.

49. Marques MAS, Bizarri CHB, Cardoso, JN, Aquino Neto FR. Effect of finasteride on the urinary steroid profile: a case study. In: Schänzer W, Geyer H, Gotzmann A, Mareck-Engelke U, editors. Proceedings of the 17th Cologne Workshop on Dope Analysis. Köln: Sport und Buch Strau $\beta$ 1999;317-22.

50. Horning S, Geyer H, Flenker U, Shanzer W. Detection of exogenous steroids by $13 \mathrm{C} / 12 \mathrm{C}$ analysis. In: Schänzer W, Geyer H, Gotzmann A, Mareck-Engelke U, editors. Proceedings of the 15th Cologne Workshop on Dope Analysis. Köln: Sport und Buch Strauß 1998;135-48.

51. Geyer H, Schänzer W, Mareck-Engelke U, Donike M. Factors influencing the steroid profile. In: Donike M, Geyer H, Gotzman A, Marek-Engelke U, Rauth S, editors. Proceedings of the 15th Cologne Workshop on Dope Analysis. Köln: Sport und Buch Strauß 1998;129-33.

52. Karila T, Kosunen V, Leinonen A. High doses of alcohol increase the urinary testosterone-epitestosterone ratio in females. J Chromatogr Biomed Appl B 1996;687:109-16.

53. Nolteernsting E, Schänzer W, Donike M. Experiences with d3-testosterone/ d3-epitestosterone as internal standards for screening and confirmation. In: Donike M, Geyer H, Gotzman A, Marek-Engelke U, Rauth $\mathrm{S}$, editors. Proceedings of the 13th Cologne Workshop on Dope Analysis. Köln: Sport und Buch Strauß 1996;191-9.

54. Debruyckere G, Van Petheghem CH, De Brabander H, Debackere M. Gas chromatographic-mass spectrometric confirmation of 19-nortestosterone in the urine of untreated boars - Effect of the administration of laurabolin. Vet Q 1990;12:246-50.

55. Hemmersbach P, Hagensen AH, Misund J. Determination of urinary norandrosterone excretion in females during one menstrual cycle by gas chromatography/mass spectrometry. In: Schänzer W, Geyer H, Gotzmann
A, Mareck-Engelke U, editors. Proceedings of the 14th Cologne Workshop on Dope Analysis. Köln: Sport und Buch Strauß 2000;141-54.

56. Machnik M, Delahaut P, Horning S, Schänzer W. Purification and concentration of anabolic steroids by immune affinity chromatography. In: Schänzer W, Geyer H, Gotzmann A, Mareck-Engelke U, editors. Proceedings of the 14th Cologne Workshop on Dope Analysis. Köln: Sport und Buch Strau $\beta$ 1996;223-37.

57. Loh YP, Loriaux LL. Adrenocorticotropic hormone, b-lipoprotein, and endorphin-related peptides in health and disease. JAMA 1982;247:10334.

58. Paul DP, Gallant S, Orme-Johnson NR, Orme-Johnson WH, Brownie AC. Temperature dependence of cholesterol binding of cytochrome P$450 \mathrm{scc}$ of the rat adrenal. Effect of adrenocorticotropic hormone and cycloheximide. J Biol Chem 1976;251:7120-6.

59. Segura J, de la Torre R, Pascual JA, Ventura R, Farré M, Camí J. Antidoping control laboratory at the games of the XXV Olympiad Barcelona'92. Part II strategies and results. In: Donike M, Geyer H, Gotzmann, A, editors. Proceedings of the 12th Cologne Workshop on Dope Analysis. Köln: Sport und Buch Strau $\beta$ 1999;413-32.

60. Phillips GB. Relationship between serum dehydroepiandrosterone sulfate, androstenedione, and sex hormones in men and women. Eur J Endocrinol 1996;134:201-6.

61. Reed AH. Influence of statistical method used on the resulting of normal range. Clin Chem 1971;17:275-84.

62. De La Torre R, de La Torre X, Segura J, Smeyers MT, Ventura R, Torres JM, Alía C, et al. Urine contamination by microorganisms and alterations in the endogenous steroid profile. A prospective study. In: Schänzer W, Geyer H, Gotzmann A, Mareck-Engelke U, editors. Proceedings of the 16th Cologne Workshop on Dope Analysis. Köln: Sport und Buch Strau $\beta$ 1999;223-36.

63. Augensten WM. Applied gas chromatography coupled to isotope ratio mass spectrometry. J Chromatogr A 1999;852:351-71.

64. De la torre X, Gonzalez JC, Pichini S, Pascual JA, Segura J. ${ }^{13} \mathrm{C} /{ }^{12} \mathrm{C}$ isotopic ratio MS analysis of testosterone, in chemicals and pharmaceutical preparations. J Pharm Biomed Anal 2001;24:645-50.

65. Cayet C, Lichtfouse E. ${ }^{13} \mathrm{C}$ of plant-derived n-alkanes in soil particlesize fractions. Organic Geochem 2001;32:253-8.

66. Eggers T. You are what you eat... or are you? Tree 2000;15:265-6.

67. Mathurin JC, Ferry M, Bougogne E, Barrault Y, Ceaurriz J. Differenciation de l'origine exogene ou endogene de produits dopants par l'analyse isotopique du carbone. Rev Franc des Lab 2001;331:29-36.

68. Laure P. Conduites dopantes: quelques modalites de consommation dês produits. Rev Franc des Lab 2001;331:25-8.

69. Welzel P, Hobert K, Ponty A. 4-androsten-3-17-one from cholesterol, $\beta$ sitosterol, and campesterol by chemical degradation. Tetra Let 1983;24: 3199-202. 